\title{
Optimización de los costos de muestreo en la manufactura de shampoo y acondicionador
}

\author{
María del Carmen Rosas Guerrero* \\ Tecnológico de Monterrey, Querétaro, México \\ Emilio Pérez Pacheco* \\ Instituto Tecnológico Superior de Calkiní. Campeche, México \\ Recibido: 14 de mayo del 2018 / Aprobado: 26 de junio del 2018 \\ doi: 10.26439/ing.ind2018.n036.2452
}

\begin{abstract}
Resumen: Optimizar los costos en el proceso de muestreo en la producción de shampoo y acondicionador a través del diseño bibliográfico, debido a trabajos previos de investigación en temas de producción, muestreo y calidad en diferentes ramas industriales entre los años 2010-2018, y de campo, por los datos recolectados en una empresa de giro cosmético situada en Bajío, México, y los análisis de las muestras a través de dos fases: financiera y calidad.
\end{abstract}

Palabras clave: costos de producción / muestreo (estadística) / procesos de manufactura / control de costos

\section{Optimization of sampling costs in shampoo and conditioner manufacturing}

Abstract: Cost optimization in the sampling process of shampoo and conditioner manufacturing by the bibliographic design, using previous research on production, sampling and quality in different industrial branches between the years 2010 and 2018, and by the field design, using data collected in a beauty product company located in Bajío, Mexico. Sample analysis through the financial and quality phases.

Keywords: production costs / sampling (statistics) / manufacturing processes / cost control

* Correos electrónicos: shamu1214@hotmail.com, eperez@itescam.edu.mx 


\section{INTRODUCCIÓN}

Actualmente, las empresas de manufactura están buscando opciones para reducir costos y que se obtengan mayores ganancias. Se sabe que implementar alternativas requiere de un análisis de los procesos actuales. Por lo tanto, esta investigación se enfoca en mostrar una alternativa viable para optimizar costos, específicamente en la fuente de scrap dentro de la industria de cosméticos.

Siguiendo con el análisis de scrap, se tienen varias fuentes que generan esta pérdida; una de ellas es el producto que se pierde debido a la toma de muestras por parte del área de calidad para ser analizado y que sirve de fundamento para la liberación de producto terminado. Cuando una botella es tomada para ser analizada por el laboratorio de calidad, es necesario retirar la tapa, por lo que, en ese momento, no se puede vender a cliente, ya que el empaque fue alterado y podría tener partículas extrañas en su contenido, como el polvo. Además que, al extraer producto de granel para ser analizado, el peso de la botella es modificado, es decir, la botella ya no cumpliría con la cantidad que es ofrecida a cliente a través de su etiqueta.

Las empresas del sector industrial mencionado basan el proceso de liberación de producto terminado en muestreos aleatorios de la producción, es decir, utilizan datos estadísticos de acuerdo con el número de unidades producidas por cada lote. Estos datos estadísticos son de acuerdo con las tablas Militar Estándar (MIL-STD) (Vega Rangel, 2016). Se toma la muestra que indican las tablas y se llevan al laboratorio para los análisis químicos y para otras pruebas. Entre mayor cantidad de productos se elaboren por lote, mayor es el número de muestras como lo indican las tablas Militar Estándar, ya que no toma en cuenta la mejoras o la estabilidad en los procesos de producción. Se toman las muestras necesarias y se analizan realizando pruebas destructivas, es decir, que el producto muestreado no se puede recuperar para su posterior venta. Esta situación resulta en una mayor pérdida para las empresas, ya que se están pagando el costo de manufactura y no hay posibilidades de recuperar este costo en esa pieza.

Por tal razón, esta investigación propone una alternativa para la reducción de costos que se incurren al muestrear los lotes de producción en las empresas de manufactura de shampoo y acondicionador a través de una validación que consiste en establecer evidencia documentada 
que proporcione seguridad de que un proceso cumpla con las especificaciones y características de calidad predeterminadas. Esta validación es el soporte para la liberación de producto terminado y así poder optimizar el número de muestras por cada lote de producción en la industria de shampoo y acondicionador. Para explicarlo, es necesario conocer el costo a optimizar y las variables críticas del granel tanto en fabricación como empacado.

Esta investigación está enfocada en la región geográfica llamada 'Bajío' dentro de territorio mexicano. Esta región está localizada justo en el centro del país, dentro del triángulo industrial de las tres ciudades más grandes de México: Ciudad de México, Guadalajara y Monterrey. La región Bajío comprende un radio de $400 \mathrm{~km}$, y abarca el 70 \% del establecimiento industrial, donde cubre los estados de Aguascalientes, Jalisco, Guanajuato, San Luis Potosí y Querétaro (Pilotzi, 2018).

\section{MÉTODO}

\subsection{Marco teórico}

En la actualidad, la industria de cosméticos se encarga de la elaboración de productos tales como shampoo, acondicionadores, tintes, jabones para cuerpo, entre otros (Salud, 1995). Dichos productos deben cumplir con estrictas normas de calidad, previo a su comercialización. Las empresas de este sector industrial se basan, principalmente, en muestreos aleatorios de la producción, es decir, utilizan datos estadísticos de acuerdo con el número de unidades producidas por cada lote. Estos datos estadísticos son de acuerdo con las tablas Militar Estándar (MIL-STD) (Vega Rangel, 2016). Se toma una muestra y se lleva al laboratorio para los análisis químicos y para otras pruebas, entre las que se encuentran las pruebas destructivas, es decir, que el producto muestreado no se puede recuperar para su posterior venta. Entre mayor cantidad de productos se elaboren por lote, es mayor el número de muestras, lo que resulta en una pérdida para la empresa.

La presente investigación propone una alternativa para la reducción de costos que se incurren al muestrear los lotes de producción en las empresas de manufactura de shampoo y acondicionador en la zona de Bajío. 


\subsection{Crecimiento del sector cosmético en México}

El sector del cuidado personal está conformado por diferentes categorías según la Procuraduría Federal del Consumidor (Profeco): 1) productos para el cabello donde se incluye el shampoo, acondicionador, enjuague, spray, mousse, fijador, geles, cremas para peinar, tratamiento capilares, tintes; 2) productos para la piel que son los repelentes de insectos; 3) productos para la higiene como las cremas y espumas para rasurar, máquinas de afeitar y repuestos; 4) producto para uñas y aquellos que buscan modificar los olores corporales como desodorantes, fragancias, perfumes y talcos; y 5) maquillaje (Silva, 2012).

El sector de shampoo y acondicionador es de los más rentables y de mayor crecimiento desde el 2010. Según la Cámara Nacional de la Industria de Productos Cosméticos (Canipec) de México, los productos para el cuidado del cabello representaron el 19,4 \% del valor de ventas de toda la industria del cuidado personal en el 2014 y crecieron un $5 \%$ el 2015 (Moguel, 2014).

El sector industrial de la zona de Bajío se encuentra en crecimiento durante los últimos cinco años, y es el factor importante en el crecimiento del producto bruto interno (PBI) (Martínez, 2015). En esta investigación, el sector industrial de shampoo y acondicionador de dicha zona es el actor principal en la investigación, ya que sus finanzas se ven impactadas por la toma de muestras de producto terminado en cada lote de producción.

En la industria de shampoo, existen varios tamaños para satisfacer cualquier necesidad o preferencia del consumidor. El shampoo puede producirse desde $7,5 \mathrm{ml}$, que por lo general se ofrece como muestra gratis dentro de revistas, hasta $1200 \mathrm{ml}$, que es un tamaño familiar. Dentro de este rango, se tienen tamaños para todo tipo de necesidades. El más comprado por los clientes dentro del territorio mexicano es la botella de $400 \mathrm{ml}$ (Sánchez, 2017) debido a:

i) Todas las empresas fabrican este tamaño y ofrecen una oferta equitativa a los posibles clientes.

ii) El promedio de cantidad usada por cada baño es de $17 \mathrm{ml}$ para mujeres, con un uso mensual de una botella de $400 \mathrm{ml}$ por mes aproximadamente.

iii) El promedio de cantidad usada por cada baño es de $8 \mathrm{ml}$ para hombres, que terminan una botella de $400 \mathrm{ml}$ cada dos meses. 
Referente a la producción de acondicionador, la tendencia de los clientes es buscar una botella que haga juego con el shampoo, es decir, busca la sincronía en tamaños. Por esta razón, también las botellas de $400 \mathrm{ml}$ son las más vendidas.

Para fines de esta investigación, los análisis que se muestran en el documento se basan en una manufactura de tamaños de $400 \mathrm{ml}$ por la razón explicada anteriormente. Con esto, se puede conocer el mayor impacto financiero que tienen las empresas.

\subsection{Fabricación del granel del shampoo}

Para la producción de shampoo se necesitan varios materiales, pero los que tienen mayor porcentaje dentro de la fórmula total son:

i) Surfactante

ii) Agua

iii) Salmuera

iv) Ingredientes menores

El surfactante es un elemento que actúa como detergente que permite reducir la tensión superficial que existe en un fluido. También se le conoce como tensioactivo o tensoactivo; se componen por partes hidrófobas, es decir, que rechaza el agua, y por partes hidrófilas, que son solubles en agua. Esta interacción de las dos partes hace que se generen burbujas; sin embargo, las burbujas no son las encargadas de ofrecer limpieza, sino la tensión superficial (Sánchez, 2017). En la fabricación de shampoo, el surfactante representa cerca del $60 \%$ del total de ingredientes, y es el componente más importante y mayoritario, tanto en desempeño como en cantidad.

El agua sirve para darle consistencia al surfactante y para tener el volumen que se requiere: la mezcla entre el agua y el surfactante es vital para lograr la red cristalina. La formación de esta red cristalina es el principal proceso en la fabricación de shampoo, ya que su función principal es asegurar la mezcla homogénea entre las fases que conforman el producto y que no se visualicen dos fases en la botella debido a las partes hidrófobas y las hidrófilas. Para lograr esta red se tiene dos variables muy importantes: el tiempo en el proceso y la temperatura. En la fabricación de shampoo el surfactante representa cerca del $25 \%$ del total de ingredientes. La salmuera es una concentración de agua 
con sal con la finalidad de mantener la conservación del shampoo y que se cumpla con la fecha de caducidad. La salmuera representa un $10 \%$ del total de los ingredientes. Los ingredientes menores representan los ingredientes que están por debajo del $5 \%$ del total.

La fabricación del shampoo se basa en tres procesos:

i) Proceso caliente

ii) Freezeout

iii) Proceso frío

El proceso caliente es un tanque de 12 toneladas que hace la mezcla del surfactante, del agua y de la salmuera. Este proceso está en continuo movimiento hasta que se tiene una mezcla no homogénea por las propiedades del surfactante. Es difícil conseguir una mezcla homogénea que se mantenga a lo largo de la fecha de caducidad.

Cuando se logran mezclar los ingredientes mencionados y el tiempo establecido, se pasa al proceso de freezeout. En este proceso se genera la red cristalina. Esta red se logra al pasar el granel almacenado en el proceso caliente por una tubería en un determinado tiempo para que llegue al segundo tanque de almacenamiento a una temperatura más baja. El freezeout no puede variar su tiempo, es decir, el tiempo que quedó estipulado en la validación; siempre será el mismo: no puede variar porque podría impactar en la fecha de caducidad y no mantener el mismo desempeño o apariencia en el momento de la manufactura. Para lograr bajar la temperatura entre los dos tanques, es necesario contar con una tubería adecuada de acero inoxidable que no permita el almacenamiento de producto y genere posibles microorganismos. Este proceso es el más crítico, ya que también asegura que el shampoo sea capaz de limpiar las impurezas del cabello y del cuero cabelludo del consumidor. De no contar con el tiempo y la temperatura correctos, podrían verse dos fases en la botella, una del agua con sus ingredientes menores y otra fase con el surfactante, que da como resultado que en la fase de agua no se cumpla con la función de limpiar.

El tercer proceso se lleva a cabo en un tanque de almacenamiento de la misma capacidad que el proceso en caliente, es decir, en el tanque de 12 t. Empieza el proceso cuando se recibe el granel después de pasar por el freezeout a una temperatura más baja para que empiece su mezcla con los ingredientes menores, es decir, en esta etapa se adicionan los últimos materiales para completar la fórmula. Normalmente estas 
adiciones son las que aseguran la estética del producto. Cuando llega el granel a este tanque es cuando se asegura que el proceso de la creación de la red cristalina ha sido completado correctamente, ya que las adiciones menores se integran para seguir con el granel homogéneo. Este proceso debe asegurar la temperatura constante durante el tiempo que dure el proceso, es decir, debe mantenerse por debajo de los $40{ }^{\circ} \mathrm{C}$ para asegurar la mezcla homogénea. La figura 1 muestra el freezeout como el enlace entre los dos tanques de proceso para asegurar la red cristalina.

Con el proceso en frío completo, se procede a transferirlo al área de empaque.

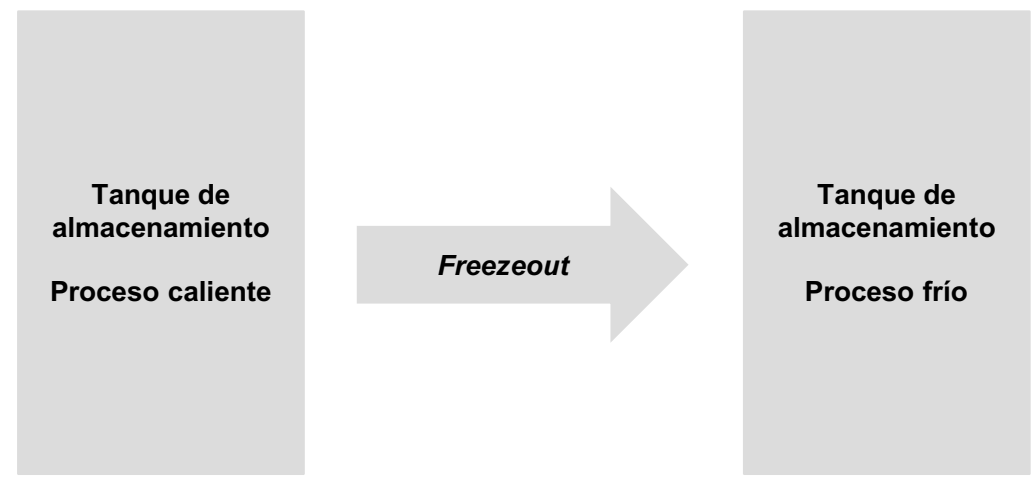

Figura 1. Proceso de fabricación de shampoo

Elaboración propia

\subsection{Fabricación del granel del acondicionador}

Para la producción de acondicionador se necesitan varios materiales, pero los que tienen mayor porcentaje dentro de la fórmula total son:

i) Agua

ii) Alcoholes

iii) Siliconas

iv) Surfactante

v) Ingredientes menores

El agua es el ingrediente con mayor porcentaje: un $93 \%$ del total de ingredientes. El agua es primordial para que pueda reaccionar con los alcoholes y la silicona y permitir un cabello suave y sedoso al momento de peinar. 
Se tienen adiciones de alcoholes para que hagan una mezcla homogénea con el agua y permitir su conservación. El principal alcohol es el alcohol cetílico, que es un alcohol graso que se utiliza en la industria cosmética para que el cabello reseco pueda recuperar su consistencia habitual de brillo. En la fabricación de acondicionador, el alcohol cetílico representa el $3 \%$ del total de ingredientes.

Las siliconas son un ingrediente importante para la fabricación del acondicionador, ya que son las que recubren el cabello para darle brillo y protegerlo de factores externos como la humedad, luz solar, contaminación, entre otros. Los ingredientes menores representan el $1 \%$ del total de la fórmula.

La fabricación del acondicionador se basa en tres procesos:

i) Proceso caliente

ii) Primer freezeout

iii) Segundo freezeout

Los tres procesos se llevan a cabo en un solo tanque de almacenamiento. Para fines de esta investigación, se basará el volumen en $12 \mathrm{t}$, por lo que se adiciona el agua correspondiente a esta cantidad con los alcoholes, siliconas y surfactante necesarios para empezar la mezcla. Este proceso está en continuo movimiento hasta lograr una sola fase en todo el tanque sin identificar los diferentes ingredientes que lo componen. Comúnmente se asegura una temperatura constante en todo el tanque mayor a $70{ }^{\circ} \mathrm{C}$.

Cuando se logra la mezcla homogénea, se empieza el proceso del primer freezeout en donde se baja la temperatura de todo el contenido del tanque a $50{ }^{\circ} \mathrm{C}$ para que se siga mezclando y se conserven las propiedades del proceso en caliente. Esta disminución de la temperatura se logra a través de una recirculación de una tubería en el mismo tanque: no es necesario transferirlo a otro; sin embargo, algunas empresas prefieren contar con dos o tres tanques de almacenamiento para asegurar la temperatura, ya que no cuentan con máquinas con gran exactitud.

Cuando se logra la temperatura requerida, se empieza el segundo freezeout en donde se baja nuevamente la temperatura a menos de $40{ }^{\circ} \mathrm{C}$. Se adicionan los ingredientes menores durante este proceso de enfriamiento mezclándolos hasta tener una sola fase en todo el granel del tanque de almacenamiento. Durante este segundo freezeout se debe 
asegurar la temperatura constante durante el tiempo que dure el proceso, es decir, debe mantenerse por debajo de los $40{ }^{\circ} \mathrm{C}$ hasta el momento de la transferencia. Con el proceso completo, es decir, cuando se logra una mezcla homogénea hasta con los ingredientes menores y se tiene la temperatura menor a $40{ }^{\circ} \mathrm{C}$, se procede a transferirlo al área de empaque.

La figura 2 muestra que un solo tanque de almacenamiento es necesario para realizar los cambios de temperatura.

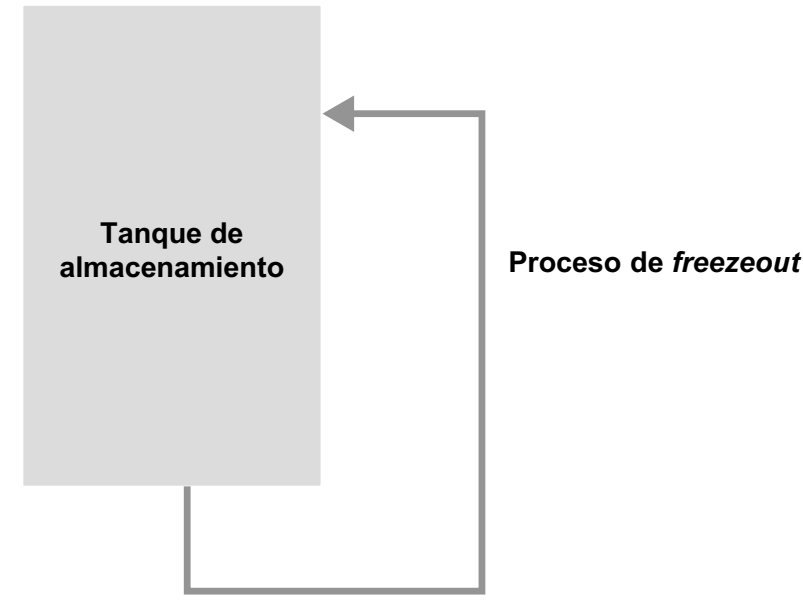

Figura 2. Proceso de fabricación de acondicionador Elaboración propia

\subsection{Proceso de empaque}

El área de empaque tiene los siguientes procesos:

i) Clasificación de materiales

ii) Llenado

iii) Tapado

iv) Etiquetado

v) Empacado en caja

vi) Emplayado

La clasificación de materiales empieza cuando las botellas y tapas son introducidas en la banda transportadora de la línea de producción. El proceso de llenado es la transferencia del granel a la llenadora y empieza 
a vaciarse a una velocidad que no permite tener esfuerzos mecánicos adicionales. Este proceso es el responsable de que el volumen o peso se cumpla con lo que está mostrado en la etiqueta; la llenadora tiene boquillas que entran al cuello de la botella e introducen el granel necesario sin derrames. Después de que la botella se llene, se tapa y es posicionada para cerrar con un torque en específico y no pueda derramarse el granel con el empacado y el transporte. La tapa tiene que embonar con el cuello de botella para poder cerrarse. Este proceso pasa sobre la línea para que pueda ser un proceso continuo. Una vez que se tiene la botella cerrada para evitar una posible contaminación de partículas extrañas, sigue su transporte sobre la banda transportadora para llegar a la etiquetadora, donde las dos etiquetas son pegadas. La etiqueta frontal es la que muestra el nombre del producto, si es shampoo o acondicionador, el claim, el arte y la cantidad ofrecida al consumidor. La etiqueta trasera muestra los ingredientes, la forma de uso, los registros sanitarios, código de barras y dónde fue fabricado el producto. El proceso de empaque puede ser manual o automático; si es manual, se tiene que capacitar a los empleados a acomodar las botellas para que no se golpeen entre ellas y que las tapas no se abran durante el transporte a los centros de distribución; si es automático, se programa el robot para que contabilice el número de botellas correctas y las posicione dentro del corrugado. Al tener el formado de la caja completa, se espera al final de la banda transportadora que lleguen más cajas y así poder envolver la tarima completa con una película de plástico para evitar que el producto se caiga.

\subsection{Muestreo en la línea de producción}

En el proceso de toma de muestras, los operadores están capacitados para decidir cuántas botellas tomar durante el lote de producción. Este número se basa en las tablas MIL-STD en un muestreo por atributos, ya que las botellas se dividen en defectuosas y en no defectuosas. Se toma un Nivel General de Inspección II como política de la empresa; no se elige como I porque la discriminación de defectos no se acepta como baja y no se elige como nivel III debido a que el costo de la muestra es muy alto porque algunas de las pruebas son destructivas en el laboratorio. Como ya son productos vendibles al consumidor y se tienen más de cinco lotes consecutivos liberados a cliente por cada versión, se puede establecer una inspección normal con un nivel de calidad aceptable (NCA) de $0 \%$ para defectos críticos y 1,5\% para defectos encontrados en las 
botellas debido a lo permitido por el gobierno mexicano referente al llenado de granel estipulado en la etiqueta. Tomando el ejemplo de una botella de shampoo y acondicionador de $400 \mathrm{ml}$ y con un lote de producción de 12 t y una densidad de 0,98 del granel de shampoo más vendido, el peso mínimo de la botella es de $392 \mathrm{~g}$, que equivale a los $400 \mathrm{ml}$ que aparece en la etiqueta. Con estos datos, se tiene un lote de producción de 30612 botellas llenas. Este número de botellas representa 315 unidades en un nivel de inspección II. Este número de piezas se tienen que tomar de la producción para su análisis. Para llevar a cabo las pruebas físicas y químicas, se abre la tapa y, en ese instante, la botella no puede ser vendida a cliente porque se les realizan pruebas destructivas, ya que se abre la botella y el granel queda expuesto a una contaminación. Estos análisis son: $\mathrm{pH}$, alcohol cetílico, siloxano, viscosidad, gravedad específica y tamaño de partícula.

Basado en el tamaño y la versión más vendida en el mercado mexicano, es decir, $400 \mathrm{ml}$, se tiene un costo de manufactura en la empresa de este sector industrial de $\$ 6,30$ sin tomar en cuenta los gastos logísticos. Cada vez que se produce un lote de producción de $400 \mathrm{ml}$, se muestrean 315 piezas aleatoriamente durante su manufactura. Como promedio se producen 60 lotes diarios de todos los tamaños, de los cuales 18 lotes son de $400 \mathrm{ml}$. Es decir, se tiene un scrap de 28350 botellas a la semana, que representan $\$ 178,605 \mathrm{MXN}$ por cada lote de producción como pérdida semanal para la empresa. Este monto es la pérdida que representa a la empresa, ya que las unidades son destinadas a scrap por ser abiertas.

\subsection{Validación}

El proceso de validación es importante cuando existe alguna modificación, cambio o nuevo producto en una planta manufacturera a través de estudios $R \& R$ (repetitividad y reproducibilidad). Los estudios R\&R analizan la variación entre el método de medición y las distintas personas e instrumentos de medición que pueden realizar dichas mediciones. El objetivo principal es reducir o controlar la variabilidad de la medición de un proceso o máquina mediante el estudio de los posibles motivos de variabilidad para conseguir la mayor exactitud de medición en el proceso al disminuir producto fuera de especificación. Cada empresa tiene su propio proceso de validación; sin embargo, se recomienda contar con al menos tres corridas de producción con diferentes cuadrillas de operadores. 


\subsection{Diseño de la investigación}

La estrategia de esta investigación se basa en un diseño bibliográfico y de campo. Es un diseño bibliográfico por la investigación de trabajos anteriores realizados en el tema de reducción de costos en un ambiente industrial y en los requerimientos necesarios para asegurar el proceso productivo dentro de estándares de calidad a lo largo del tiempo. También es un diseño de campo por la investigación del impacto de las variables en el proceso de costos, al igual que los costos de producción en la industria de shampoo y acondicionador.

Se tienen dos etapas:

i) Financiera

ii) Calidad

La primera etapa consiste en obtener la información de costos de los lotes de producción con sus respectivas pérdidas debido al proceso de scrap; para lograr este resultado, es necesario conocer la gama de productos de mayor volumen de venta con sus costos respectivos para lograr mostrar la optimización de los costos de muestreo en una empresa de shampoo y acondicionador. Esto es con la finalidad de que cada empresa pueda proyectar la optimización de sus costos con esta propuesta.

La segunda etapa consiste en, primero, conocer cómo se lleva a cabo el proceso de R\&R, cuáles son sus condiciones, sus pasos y sus criterios de éxito para asegurar que el proceso productivo sea repetible y reproducible, además de obtener los resultados de las variables críticas de las muestras elegidas. Estos análisis se harán después de que se compruebe que el proceso esté en control estadístico, es decir, que todo el proceso haya sido validado.

Esta investigación identifica su población finita que delimita a las empresas manufactureras de shampoo y acondicionador en la zona Bajío, ya que los costos y variables de calidad están enfocados en este giro industrial. La muestra probabilística es de tipo estratificada, ya que los productos se clasifican en versiones de producción y en tamaño en mililitros.

La investigación toma la población de una de las grandes empresas que tiene la región Bajío, la cual produce botellas para la venta en México y la muestra es de cinco lotes de producción de las versiones con mayor costo de producción en México de shampoo y acondicionador. La 
propuesta mostrada en esta investigación es analizar con pruebas de laboratorio solamente tres botellas por lote más la botella que se queda resguardada en las instalaciones de la empresa para futuras investigaciones internas o de gobierno, por lo que se tiene una muestra total de cuarenta botellas (20 de shampoo y 20 de acondicionador); sin embargo, solamente se tienen resultados de treinta botellas. Para seleccionar la muestra, es necesario confirmar que el producto haya sido validado en el ambiente real de producción, es decir, que sean de producciones que tuvieron un proceso de $R \& R$ para confirmar que el proceso es estable.

\section{RESULTADOS}

Los resultados de esta investigación se lograron basándose en un proceso detallado que involucra el estudio de las variables que conforman la producción de shampoo y acondicionador. Para el análisis de datos, se llevó a cabo de manera similar a como lo indica el investigador Lance Coleman en el año 2015, es decir, se aseguró de llevar a cabo las tres fases que él propone: i) leer y entender los tipos de gráficas y tablas que tiene el proceso, ii) identificar posibles tendencias o resultados, y iii) entender la capacidad del proceso instalado (Coleman, 2015).

La primera etapa consiste en la recolección de información de la planta de producción seleccionada en la región Bajío. La información fue dividida en varias fases a través de entrevistas con la gerente de calidad y con la gerente de producción, las que nos compartieron los datos necesarios para el posterior análisis. El resultado está segmentado en:

i) Productos activos

ii) Cantidad de producción

iii) Costo de manufactura

iv) Unidades a muestrear

Para la etapa de calidad, esta consiste en llevar a cabo el proceso de repetividad y reproducibilidad (R\&R) para poder validar el proceso de medición tanto para shampoo y acondicionador y ver si cumplen con lo estipulado por el equipo de calidad. El análisis de esta etapa está divido en:

i) Variables críticas de proceso

ii) Estudios R\&R 
iii) Muestreo con la reducción de muestras

iv) Análisis estadístico de variables

Los resultados que se muestran demuestran la factibilidad de optimizar costos por la reducción de muestras que se toma en cada lote de producción. Es decir, se explica la comparación de la situación actual con la propuesta de esta investigación. La situación actual que tiene la empresa de shampoos y acondicionadores es tomar el número de muestras según la recomendación de las tablas MIL-STD y la propuesta de esta investigación es tomar solamente tres piezas de producto terminado para confirmar las características críticas que aseguran el desempeño del producto con el consumidor final. Esta reducción en el número de botellas se basa en tomar las botellas como producto terminado una vez que se hayan controlado y registrado las variables críticas durante el proceso de producción. Para la toma de muestra durante el proceso de fabricación, se tiene un NCA de $0 \%$, ya que, al obtener una muestra fuera de especificación, demuestra que no se manufacturó correctamente y que, al analizar el producto terminado, también estará fuera de especificación en alguna variable. Al revisar los lotes fuera de especificación que ha presentado la planta de manufactura, se comprueba que las 315 botellas presentan el mismo rango, es decir, el total de las muestras analizadas está fuera de especificación en la misma variable. Por esta razón, y que la autoridad mexicana requiere una botella de análisis y una botella para retención, es posible reducir a tres botellas solamente manteniendo el mismo NCA de $0 \%$, ya que el granel se comporta con características iguales durante el total de las botellas.

Siguiendo con el ejemplo de la fabricación de un lote de $400 \mathrm{ml}$, se obtienen 30612 botellas llenas, de las cuales se toman 315 unidades como muestreo según la recomendación de la tabla MIL-STD. Así se puede calcular el costo por muestreo en el año:

costo de manufactura/número de botellas a producir = costo por botella costo por botella $\mathrm{x}$ unidades muestreadas por lote $=$ costo de scrap por lote costo de scrap por lote $\mathrm{x}$ número de lotes anuales $=$ costo total de scrap

Con el ejemplo del lote de shampoo de $400 \mathrm{ml}$ de la versión con mayor volumen de la empresa manufacturera en la región Bajío, se tiene un costo anual en pesos mexicanos (MXN) de scrap de una sola versión de: 
$\$ 192,856 / 30612$ botellas $=\$ 6,30$

$\$ 6,30 \times 315$ unidades muestreadas $=\$ 1984,50$

$\$ 1984,50 \times 1385$ lotes anuales $=\$ 2748532,50$ MXN total de scrap

La proyección financiera anual por una sola versión de shampoo de $400 \mathrm{ml}$ es de $\$ 2748532,50 \mathrm{MXN}$ de scrap. Es decir, este monto es pagado por la empresa sin obtener un valor agregado en la cadena de suministro, ya que estas unidades (315 botellas llenas de producto) son retiradas del volumen a vender para ser analizadas en el laboratorio y, posteriormente, ser scrap.

Con los datos expuestos anteriormente, se puede calcular la optimización de los costos de muestreo en la empresa estudiada. Se sabe, como se expuso en el apartado de costo de muestreo, que se producen 18 lotes de $400 \mathrm{ml}$ diariamente, de los cuales 4 lotes (como promedio) son producidos de la versión analizada, con un costo de scrap diario de $\$ 7938 \mathrm{MXN}$.

Al hacer la optimización de la toma de muestras, se toman solamente tres botellas en cada lote producido. Para el ejemplo de la versión de 400 $\mathrm{ml}$ más vendida, se tiene un costo de $\$ 18,90$ por cada lote de producción, y al multiplicar por los 1385 lotes anuales, se tiene un total de scrap de \$ $26177 \mathrm{MXN}$. Si se compara este monto con el costo actual, se tendría una optimización anual de costos de muestreo por \$2 722356 MXN.

Siguiendo el mismo ejercicio anterior para shampoo, se tiene un ejemplo de un lote de acondicionador de $400 \mathrm{ml}$ de la versión con mayor volumen de la empresa manufacturera en la región Bajío, y se tiene un costo anual en pesos mexicanos (MXN) de scrap de una sola versión de:

$\$ 178460$ / 30769 botellas $=\$ 5,80$

$\$ 5,80 \times 315$ unidades muestreadas $=\$ 1827$

$\$ 1827 \times 620$ lotes anuales $=\$ 1132740 \mathrm{MXN}$ total de $s c r a p$

La proyección financiera anual por una sola versión de acondicionador de tamaño de $400 \mathrm{ml}$ es de $\$ 1132740 \mathrm{MXN}$ de scrap. Es decir, este monto es pagado por la empresa sin obtener un valor agregado en la cadena de suministro, ya que estas unidades (315 botellas) son retiradas del volumen a vender para ser analizadas en el laboratorio y, posteriormente, ser scrap. 
Conociendo los datos expuestos anteriormente, se puede calcular la optimización de los costos de muestreo en la empresa estudiada.

Al hacer la optimización de la toma de muestras, se toman solamente tres botellas en cada lote producido y se obtienen los siguientes resultados:

Tabla 1

Resultados de la optimización de la toma de muestras

\begin{tabular}{ccccc}
\hline Tecnología & $\begin{array}{c}\text { Costo 3 } \\
\text { botellas (MNX) }\end{array}$ & $\begin{array}{c}\text { Número de } \\
\text { lotes anuales }\end{array}$ & $\begin{array}{c}\text { Total de } \\
\text { scrap (MXN) }\end{array}$ & $\begin{array}{c}\text { Optimización de } \\
\text { costos (MXN) }\end{array}$ \\
\hline Acondicionador & $\$ 17,40$ & 620 & $\$ 10788$ & $\$ 1121952$ \\
\hline
\end{tabular}

Elaboración propia

Esta optimización de costos significa que, por cada lote de producción, se puede tener una ganancia adicional directa, ya que se pueden vender más unidades. Siguiendo el ejemplo de la versión de $400 \mathrm{ml}$, se tiene la posibilidad de 312 botellas adicionales para venta a cliente.

Basándose en las cantidades expuestas para los ejemplos anteriores, se tiene una optimización anual proyectada de costos de muestreo en dos versiones solamente, una de shampoo y otra de acondicionador, de tamaño de $400 \mathrm{ml}$ de $\$ 3844308 \mathrm{MXN}$.

El muestreo de 315 botellas representa el 1,03\% del total de lote de shampoo y 1,02\% para acondicionador. Con la propuesta de esta investigación se tiene un $0,010 \%$ de scrap por cada lote. Es decir, los materiales utilizados se aprovechan de manera más eficiente.

Para lograr la reducción de número de muestra, es necesario llevar a cabo la segunda etapa: calidad. Esta etapa es la que requiere más tiempo en campo, ya que la validación y los resultados de laboratorio se realizan dentro de las instalaciones de la empresa. Teniendo la factibilidad de producción confirmada, se planean los tres lotes de producto terminado. Estos lotes deben ser de la misma versión y consecutivos. Si se tiene un lote con una variable fuera de especificación, se debe repetir la producción de tres lotes nuevamente.

Los resultados del shampoo de una versión en específica se muestran en la figura 3.

Los resultados del acondicionador de una versión en específico se muestran en la figura 4. 


\begin{tabular}{|c|c|c|c|c|c|c|c|c|c|c|c|c|c|c|}
\hline 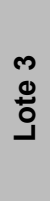 & $\begin{array}{l}00 \\
\frac{0}{0} \\
\frac{\pi}{\sigma} \\
\text { ก }\end{array}$ & 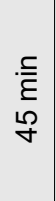 & 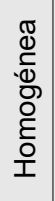 & $\begin{array}{l}\circ \\
8 \\
0 \\
0\end{array}$ & $\begin{array}{l}n \\
\frac{0}{0} \\
\frac{0}{0} \\
0 \\
\infty \\
m\end{array}$ & $\begin{array}{l}. \frac{C}{E} \\
0\end{array}$ & $\begin{array}{l}\infty \\
\frac{0}{0} \\
\frac{\pi}{0} \\
\overline{0} \\
0 \\
\dot{+}\end{array}$ & 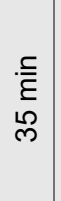 & $\begin{array}{l}\text { i̊ } \\
0 \\
0 \\
0\end{array}$ & $\frac{\circ}{0^{\circ}}$ & 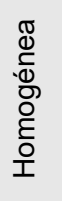 & 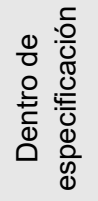 & 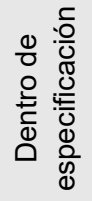 & $\begin{array}{l}\frac{\pi}{\pi} \\
\frac{\pi}{\pi} \\
\frac{\pi}{0} \\
0\end{array}$ \\
\hline دِّ & $\begin{array}{l}\text { 00 } \\
\frac{0}{0} \\
\frac{\pi}{6} \\
\text { ก }\end{array}$ & 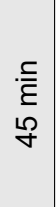 & 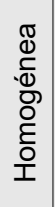 & $\begin{array}{l}\text { ○े } \\
\text { ठ } \\
0\end{array}$ & $\begin{array}{l}n \\
0 \\
\frac{0}{0} \\
\frac{0}{0} \\
\infty \\
\infty\end{array}$ & $\begin{array}{l}. \frac{\subseteq}{E} \\
0\end{array}$ & $\begin{array}{l}0 \\
0 \\
\frac{0}{0} \\
\frac{0}{6} \\
\text { ㅇ }\end{array}$ & $\begin{array}{l}. \bar{E} \\
\text { Ln } \\
\text { లn }\end{array}$ & $\begin{array}{l}\stackrel{0}{0} \\
\text { ó } \\
\text { ó }\end{array}$ & $\begin{array}{l}\stackrel{ }{N} \\
\sigma^{\prime}\end{array}$ & 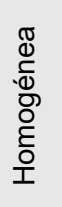 & 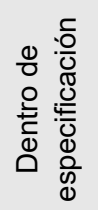 & 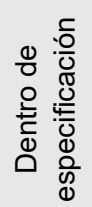 & $\begin{array}{l}\frac{\pi}{\Phi} \\
\frac{\pi}{\pi} \\
0\end{array}$ \\
\hline هِ & $\begin{array}{l}\text { D } \\
\frac{0}{0} \\
\frac{\pi}{6} \\
\text { N } \\
\text { N }\end{array}$ & $\begin{array}{l}. \frac{5}{\varepsilon} \\
\qquad f \\
q\end{array}$ & 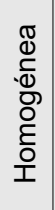 & $\begin{array}{l}\circ \\
80 \\
0 \\
0\end{array}$ & $\begin{array}{l}0 \\
0 \\
0 \\
0 \\
0 \\
0 \\
\infty \\
m\end{array}$ & $\begin{array}{l}. \frac{\subseteq}{E} \\
0\end{array}$ & $\begin{array}{l}0 \\
0 \\
0 \\
\frac{0}{0} \\
0 \\
\text { ச }\end{array}$ & 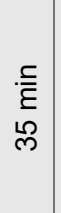 & $\begin{array}{l}\stackrel{\circ}{\circ} \\
\text { ó } \\
0^{\circ}\end{array}$ & $\begin{array}{l}\text { లి } \\
\sigma^{\prime}\end{array}$ & 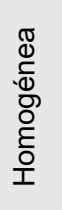 & 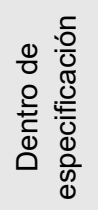 & 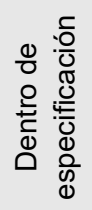 & $\begin{array}{l}\frac{\infty}{\mathbb{\pi}} \\
\frac{\pi}{\pi} \\
\frac{\pi}{0}\end{array}$ \\
\hline 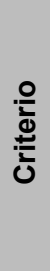 & $\begin{array}{l}0 \\
0 \\
0 \\
\frac{0}{6} \\
0 \\
0 \\
1\end{array}$ & 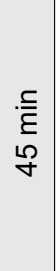 & 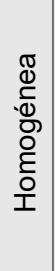 & $\frac{\sigma^{\circ}}{\frac{1}{t}}$ & $\begin{array}{l}\mathscr{0} \\
\frac{0}{0} \\
\frac{\pi}{\pi} \\
\infty \\
\infty\end{array}$ & $\begin{array}{l}. \subseteq \\
\qquad \\
\bullet\end{array}$ & $\begin{array}{l}0 \\
0 \\
\frac{0}{0} \\
\frac{0}{\pi} \\
0 \\
+ \\
+ \\
\infty \\
\infty\end{array}$ & 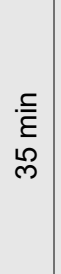 & $\frac{\delta^{\circ}}{\frac{1}{+}}$ & $\begin{array}{l}0 \\
0 \\
0 \\
1 \\
8 \\
0 \\
10\end{array}$ & 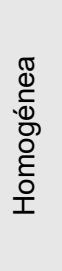 & 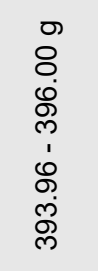 & $\begin{array}{l}\varepsilon \\
\varepsilon \\
N \\
1 \\
+\end{array}$ & 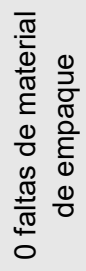 \\
\hline$\frac{\frac{0}{0}}{\frac{\frac{10}{10}}{\frac{10}{10}}}$ & 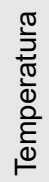 & 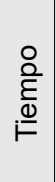 & $\begin{array}{l}\frac{\pi}{0} \\
\frac{0}{0} \\
\frac{0}{\frac{0}{\pi}} \\
\frac{0}{4}\end{array}$ & $\begin{array}{l}\mathscr{0} \\
\stackrel{0}{0} \\
\frac{0}{0} \\
\frac{0}{0} \\
\end{array}$ & 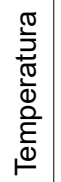 & 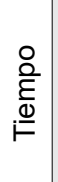 & 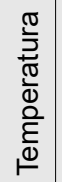 & 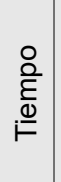 & $\begin{array}{l}0 \\
\stackrel{0}{0} \\
\frac{0}{0} \\
\frac{0}{0} \\
\frac{0}{4}\end{array}$ & I & $\begin{array}{l}\frac{\pi}{\frac{\pi}{0}} \\
\frac{\bar{\sigma}}{\frac{0}{\pi}} \\
\frac{0}{\frac{0}{4}}\end{array}$ & 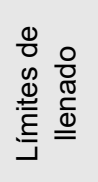 & $\begin{array}{l}\frac{0}{0} \\
\frac{\pi}{2} \\
\frac{5}{0} \\
0\end{array}$ & 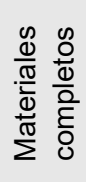 \\
\hline $\begin{array}{l}\text { 영 } \\
\text { d } \\
\text { 은 }\end{array}$ & & & $\stackrel{\oplus}{\stackrel{D}{c}}$ & & 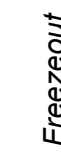 & & & & 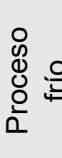 & & & $\begin{array}{l}\frac{0}{0} \\
\frac{0}{0} \\
\frac{0}{1} \\
1\end{array}$ & $\begin{array}{l}\frac{0}{0} \\
\frac{\pi}{0} \\
\frac{\pi}{2} \\
\frac{0}{ \pm} \\
\frac{\vec{w}}{4}\end{array}$ & $\begin{array}{l}\frac{0}{0} \\
\mathbb{d} \\
\mathbb{0} \\
\frac{0}{E} \\
\frac{1}{\Psi}\end{array}$ \\
\hline
\end{tabular}

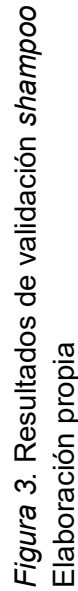




\begin{tabular}{|c|c|c|c|c|c|}
\hline Proceso & Variable & Criterio & Lote 1 & Lote 2 & Lote 3 \\
\hline \multirow{3}{*}{$\begin{array}{l}\text { Proceso } \\
\text { caliente }\end{array}$} & Temperatura & $>70$ grados & 71 grados & 71 grados & 72 grados \\
\hline & Tiempo & $45 \mathrm{~min}$ & $45 \mathrm{~min}$ & $45 \mathrm{~min}$ & $45 \mathrm{~min}$ \\
\hline & Adiciones & $+/-1 \%$ & $0,60 \%$ & $0,65 \%$ & $0,65 \%$ \\
\hline \multirow{2}{*}{$\begin{array}{c}\text { Primer } \\
\text { Freezeout }\end{array}$} & Temperatura & 50 grados & 50 grados & 50 grados & 50 grados \\
\hline & Tiempo & $15 \mathrm{~min}$ & $15 \mathrm{~min}$ & $15 \mathrm{~min}$ & $15 \mathrm{~min}$ \\
\hline \multirow{5}{*}{$\begin{array}{l}\text { Segundo } \\
\text { Freezeout }\end{array}$} & Temperatura & 38 grados & 38 grados & 38 grados & 38 grados \\
\hline & Tiempo & $15 \mathrm{~min}$ & $15 \mathrm{~min}$ & $15 \mathrm{~min}$ & $15 \mathrm{~min}$ \\
\hline & $\mathrm{pH}$ & $4.00-5.50$ & 4,30 & 4,20 & 4,30 \\
\hline & $\begin{array}{l}\text { Alcohol } \\
\text { cetílico }\end{array}$ & $2.50-4.50$ & 3,50 & 3,40 & 3,50 \\
\hline & Apariencia & Homogénea & Homogénea & Homogénea & Homogénea \\
\hline Llenado & $\begin{array}{l}\text { Límites } \\
\text { de llenado }\end{array}$ & $391.95-394.00 \mathrm{~g}$ & $\begin{array}{c}\text { Dentro de } \\
\text { especificación }\end{array}$ & $\begin{array}{c}\text { Dentro de } \\
\text { especificación }\end{array}$ & $\begin{array}{c}\text { Dentro de } \\
\text { especificación }\end{array}$ \\
\hline Etiquetado & Centrado & $+/-2 \mathrm{~mm}$ & $\begin{array}{c}\text { Dentro de } \\
\text { especificación }\end{array}$ & $\begin{array}{c}\text { Dentro de } \\
\text { especificación }\end{array}$ & $\begin{array}{c}\text { Dentro de } \\
\text { especificación }\end{array}$ \\
\hline Empacado & $\begin{array}{l}\text { Materiales } \\
\text { completos }\end{array}$ & $\begin{array}{c}0 \text { faltas de } \\
\text { material } \\
\text { de empaque }\end{array}$ & 0 faltas & 0 faltas & 0 faltas \\
\hline
\end{tabular}

Figura 4. Resultados de validación acondicionador rizos definidos Elaboración propia

Se realizó el estudio de los lotes producidos durante un año con resultados fuera de especificación en el muestreo de las 315 botellas. De los lotes producidos durante un año de las versiones elegidas, cinco de shampoo y cinco de acondicionador, se identifican 12 lotes de shampoo y 10 lotes de acondicionador que han tenido resultados fuera de objetivo, por lo que su destino final ha sido scrap. El consolidado de los resultados se muestra en la figura 5 . Se puede observar que cada variable fuera de especificación representa un proceso que no se llevó a cabo en las condiciones óptimas. Estos datos se tomaron de una investigación de los lotes producidos en el año 2017. 


\begin{tabular}{|c|c|c|c|c|c|}
\hline Tecnología & Lotes & $\begin{array}{c}\text { Variable de } \\
\text { producto terminado } \\
\text { fuera de objetivo }\end{array}$ & Límites & Resultado & $\begin{array}{c}\text { Proceso fuera } \\
\text { de } \\
\text { especificación }\end{array}$ \\
\hline \multirow{12}{*}{ Shampoo } & Lote 1 & Viscosidad & $5,000-8,000$ & 8300 & Freezout \\
\hline & Lote 2 & $\mathrm{pH}$ & $5.00-6.50$ & 6,80 & Proceso frío \\
\hline & Lote 3 & Gravedad específica & $>0.96$ & 0,95 & Proceso frío \\
\hline & Lote 4 & Viscosidad & $5,000-8,000$ & 8250 & Freezout \\
\hline & Lote 5 & $\mathrm{pH}$ & $5.00-6.50$ & 6,60 & Proceso frío \\
\hline & Lote 6 & Viscosidad & $5,000-8,000$ & 8150 & Freezout \\
\hline & Lote 7 & Viscosidad & $5,000-8,000$ & 8100 & Freezout \\
\hline & Lote 8 & Viscosidad & $5,000-8,000$ & 8100 & Freezout \\
\hline & Lote 9 & $\mathrm{pH}$ & $5.00-6.50$ & 6,6 & Proceso frío \\
\hline & Lote 10 & Límite de llenado & $393.96-396.00 \mathrm{~g}$ & 391,50 & Llenado \\
\hline & Lote 11 & Viscosidad & $5,000-8,000$ & 8200 & Freezout \\
\hline & Lote 12 & Gravedad específica & $>0.96$ & 0,95 & Proceso frío \\
\hline \multirow{10}{*}{ Acondicionador } & Lote 1 & Siloxano & $3.00-3.60$ & 3,90 & Proceso caliente \\
\hline & Lote 2 & Siloxano & $3.00-3.60$ & 3,90 & Proceso caliente \\
\hline & Lote 3 & Siloxano & $3.00-3.60$ & 3,85 & Proceso caliente \\
\hline & Lote 4 & Alcohol cetílico & $2.50-4.50$ & 4,60 & Segundo freezout \\
\hline & Lote 5 & Alcohol cetílico & $2.50-4.50$ & 4,65 & Segundo freezout \\
\hline & Lote 6 & Viscosidad & $7,500-13,000$ & 7300 & Primer freezout \\
\hline & Lote 7 & Alcohol cetílico & $2.50-4.50$ & 4,70 & Segundo freezout \\
\hline & Lote 8 & Alcohol cetílico & $2.50-4.50$ & 4,75 & Segundo freezout \\
\hline & Lote 9 & Alcohol cetílico & $2.50-4.50$ & 4,60 & Segundo freezout \\
\hline & Lote 10 & Alcohol cetílico & $2.50-4.50$ & 4,75 & Segundo freezout \\
\hline
\end{tabular}

Figura 5. Lotes fuera de especificación durante el 2017 Elaboración propia 
Al tener estos datos, se hizo un estudio de Análisis de Varianza (Anova) para confirmar que los lotes de producción se compartan de la misma manera estadísticamente, por lo que se demuestra que existe una diferencia entre las medias de los lotes de producción que se encuentran dentro de la especificación y las medias de los que se encuentran fuera de especificación en la variable de viscosidad (figura 6).
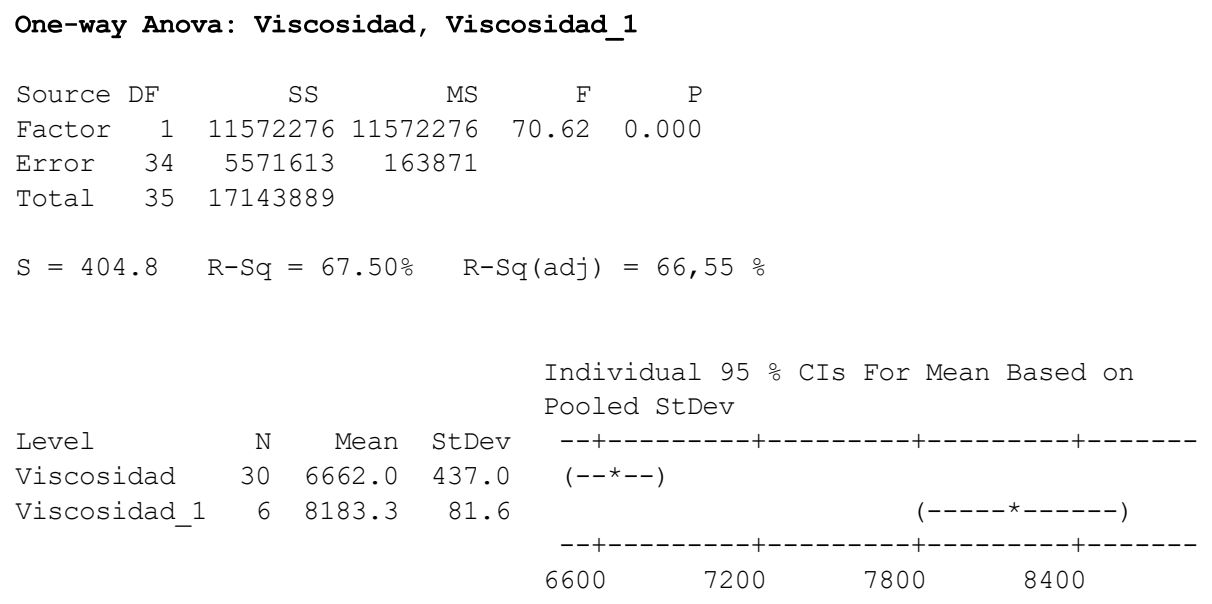

Pooled StDev $=404.8$

Figura 6. Anova lotes de viscosidad dentro y fuera de especificación Elaboración propia

También se corrió un Anova de los lotes dentro de especificación, y se contabilizaron 30 lotes del mismo producto con sus respectivos resultados de viscosidad. Para hacer esto, el total se dividió en dos grupos para que pudieran ser comparados. La figura 7 muestra que no existe una diferencia estadística entre los lotes dentro de especificación de viscosidad, ya que p-value es mayor a 0,05. Esto significa que los lotes se comportan de la misma manera en su producción.

Se corrió un Anova de los lotes fuera de especificación, aunque se tienen 6 datos solamente. Se dividió el total en dos grupos para poder analizar las diferencias. La figura 8 muestra que no existe una diferencia estadística entre los lotes fuera de especificación de viscosidad, ya que p-value es mayor a 0,05. Esto significa que los lotes se comportan de la misma manera en su producción. 

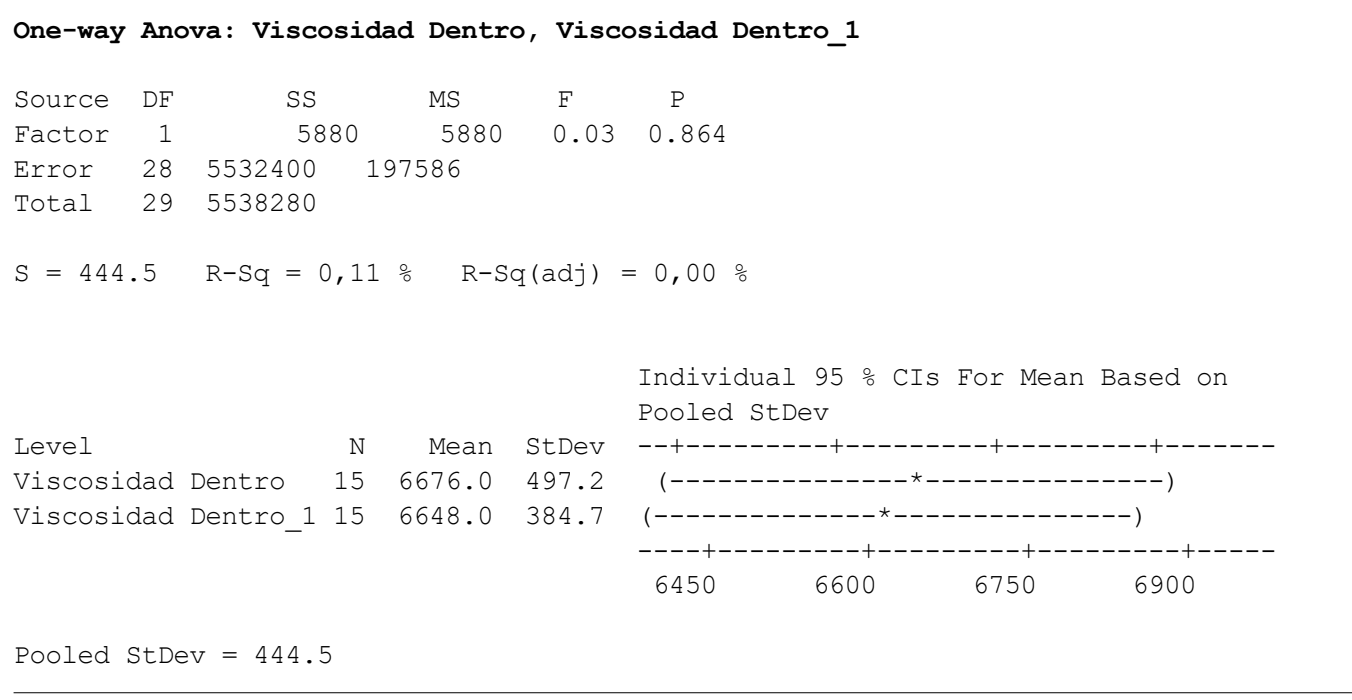

Figura 7. Anova lotes de viscosidad dentro de especificación Elaboración propia
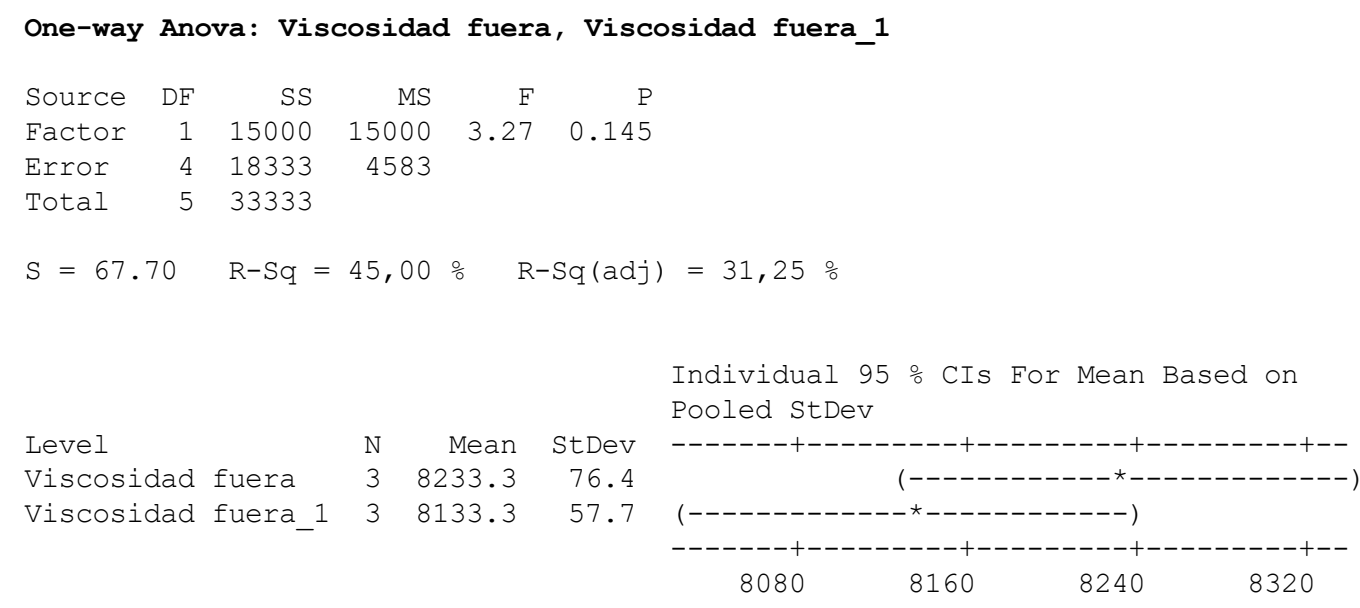

Pooled StDev $=67.7$

Figura 8. Anova lotes de viscosidad fuera de especificación Elaboración propia 
El mismo ejercicio de Anova se corrió para lotes de acondicionador que se encuentran fuera de especificación en la variable de alcohol cetílico. Se analizaron 30 lotes dentro de especificación y 6 lotes fuera de especificación de la misma variable. Se tiene un análisis de Anova comparando la variabilidad de las medias que hay entre los dos grupos. La figura 9 demuestra que existe una diferencia entre las medias de los lotes de producción que se encuentran dentro de especificación y las medias de los que se encuentran fuera de especificación en la variable de alcohol cetílico.

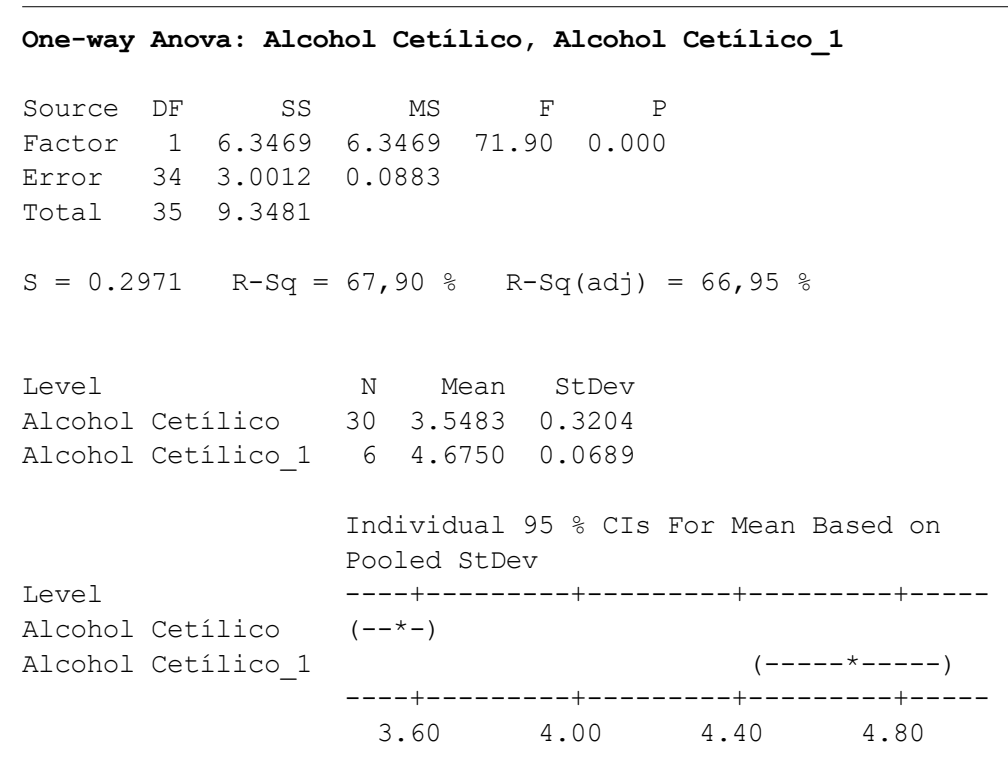

Pooled StDev $=0.2971$

Figura 9. Anova lotes de alcohol cetílico dentro y fuera de especificación Elaboración propia

También se corrió un Anova de los lotes dentro de especificación, y se contabilizaron 30 lotes del mismo producto con sus respectivos resultados de alcohol cetílico. La figura 10 muestra el resultado de la corrida de Anova, lo que demuestra que no existe una diferencia estadística entre los lotes dentro de especificación de alcohol cetílico, ya que p-value es mayor a 0,05. Esto significa que los lotes se comportan de la misma manera en su producción.

A su vez se corrió un Anova de los lotes fuera de especificación; aunque se tienen seis datos solamente, se dividió el total en dos grupos para poder analizar las diferencias. La figura 11 muestra que no existe 
una diferencia estadística entre los lotes fuera de especificación de alcohol cetílico, ya que p-value es mayor a 0,05. Esto significa que los lotes se comportan de la misma manera en su producción.

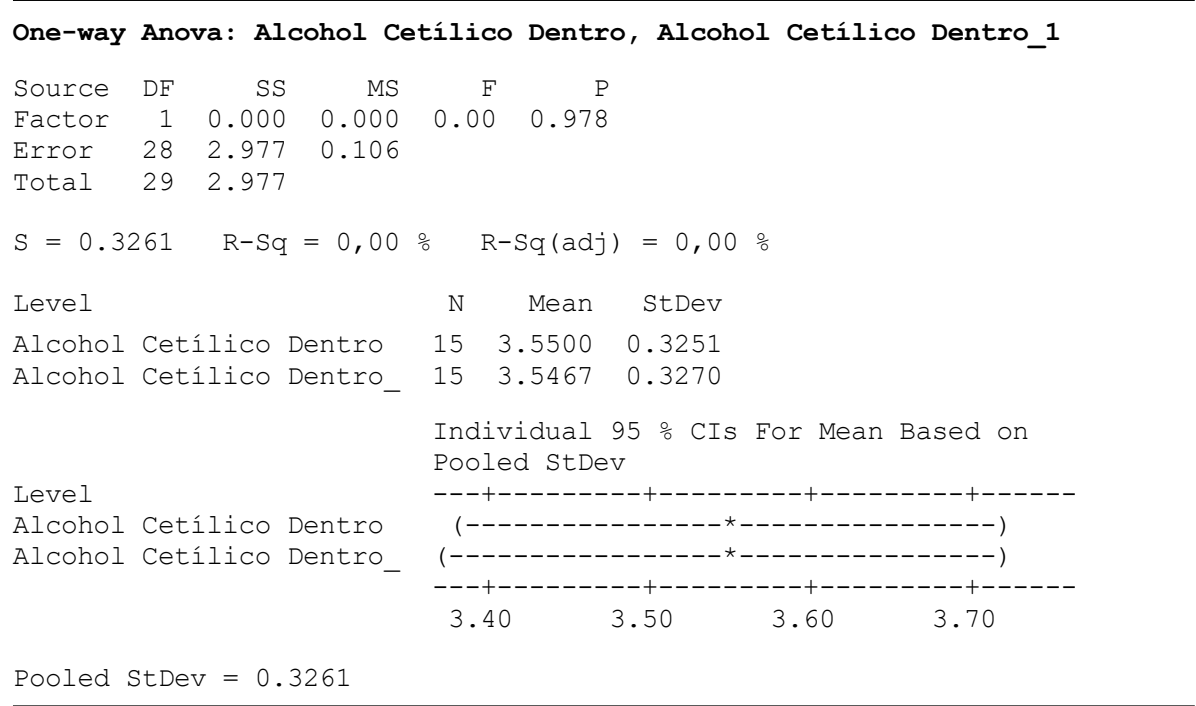

Figura 10. Anova lotes de alcohol cetílico dentro de especificación Elaboración propia

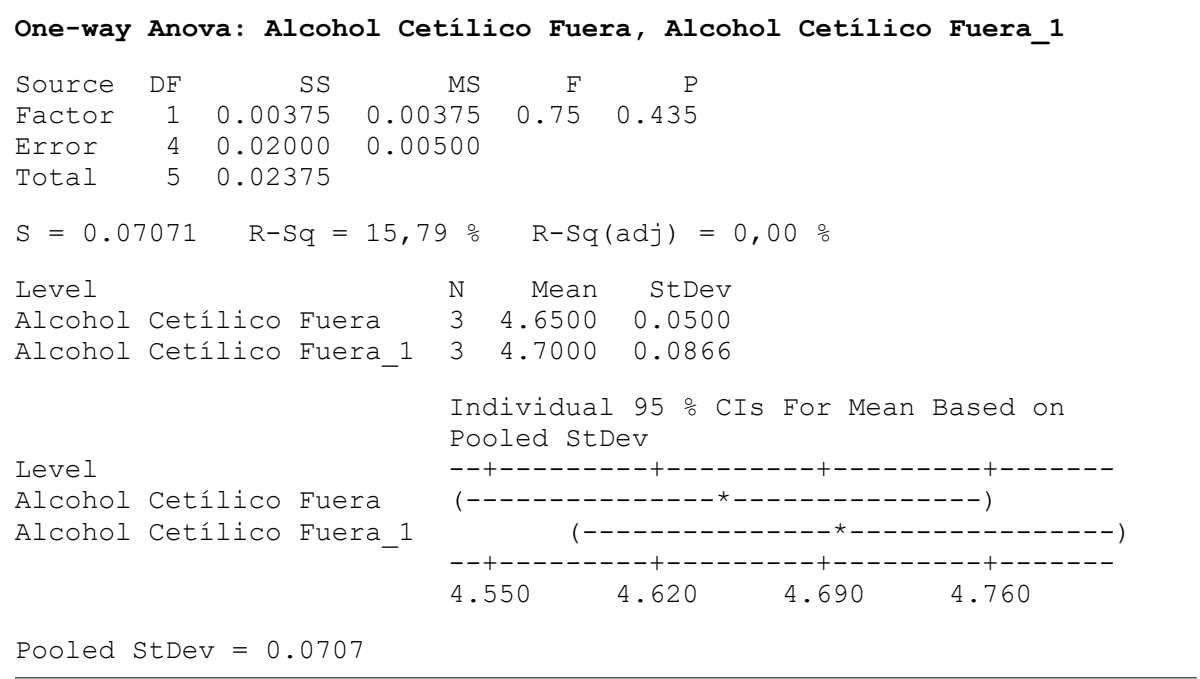

Figura 11. Anova lotes de alcohol cetílico fuera de especificación Elaboración propia 
Con estos análisis se demuestra que los lotes se comportan estadísticamente similares en cuanto a las variables seleccionadas y que, al tener una botella dentro de especificación, se tiene todo el lote de producción en las condiciones deseadas.

Conociendo los resultados mostrados en este trabajo, las variables críticas a evaluar para evitar un lote defectuoso se muestran en la figura 12 para la fabricación de shampoo y la figura 13 para el acondicionador. Estas variables tienen que ser monitoreadas en cada lote para asegurar que el desempeño del producto final se conserve.

\begin{tabular}{|c|c|c|c|}
\hline Proceso & Variable & Criterio & Momento \\
\hline \multirow{4}{*}{$\begin{array}{l}\text { Proceso } \\
\text { caliente }\end{array}$} & Temperatura & $>70$ grados & $\begin{array}{l}\text { Después del minuto } 5 \text {, } \\
\text { asegurar la temperatura }\end{array}$ \\
\hline & Tiempo & $45 \mathrm{~min}$ & \multirow{3}{*}{$\begin{array}{l}\text { Al minuto } 45 \text {, antes de } \\
\text { pasar al proceso de } \\
\text { freezeout }\end{array}$} \\
\hline & Apariencia & Homogénea & \\
\hline & Adiciones & $+/-1 \%$ & \\
\hline \multirow[b]{2}{*}{ Freezeout } & Temperatura & 38 grados & \multirow{2}{*}{$\begin{array}{l}\text { Al minuto } 6 \text {, antes de pasar } \\
\text { al segundo tanque de } \\
\text { almacenamiento }\end{array}$} \\
\hline & Tiempo & $6 \min$ & \\
\hline \multirow{5}{*}{ Proceso frío } & Temperatura & $38-40$ grados & $\begin{array}{l}\text { Durante los } 35 \text { minutos del } \\
\text { proceso frío }\end{array}$ \\
\hline & Tiempo & $35 \mathrm{~min}$ & \multirow{4}{*}{$\begin{array}{l}\text { Al minuto } 35 \text {, antes de la } \\
\text { transferencia a empaque }\end{array}$} \\
\hline & Adiciones & $+/-1 \%$ & \\
\hline & $\mathrm{pH}$ & $5,00-6,50$ & \\
\hline & Apariencia & Homogénea & \\
\hline Llenado & Límites de llenado & $393,96-396,00 \mathrm{~g}$ & Al $100 \%$ de la producción \\
\hline Etiquetado & Centrado & $+/-2 \mathrm{~mm}$ & Muestreo aleatorio \\
\hline Empacado & $\begin{array}{l}\text { Materiales } \\
\text { completos }\end{array}$ & $\begin{array}{c}0 \text { faltas de material } \\
\text { de empaque }\end{array}$ & Al $100 \%$ de la producción \\
\hline
\end{tabular}

Figura 12. Parámetros críticos en la fabricación de shampoo Elaboración propia 


\begin{tabular}{|c|c|c|c|}
\hline Proceso & Variable & Criterio & Momento \\
\hline \multirow{3}{*}{$\begin{array}{l}\text { Proceso } \\
\text { caliente }\end{array}$} & Temperatura & $>70$ grados & $\begin{array}{l}\text { Después del minuto } 5 \text {, } \\
\text { asegurar la temperatura }\end{array}$ \\
\hline & Tiempo & $45 \mathrm{~min}$ & \multirow{2}{*}{$\begin{array}{l}\text { Al minuto } 45 \text {, antes de } \\
\text { pasar al proceso del primer } \\
\text { freezeout }\end{array}$} \\
\hline & Adiciones & $+/-1 \%$ & \\
\hline \multirow{2}{*}{$\begin{array}{l}\text { Primer } \\
\text { freezeout }\end{array}$} & Temperatura & 50 grados & \multirow{2}{*}{$\begin{array}{l}\text { Al minuto } 15 \text {, antes de pasar } \\
\text { al segundo freezeout }\end{array}$} \\
\hline & Tiempo & $15 \mathrm{~min}$ & \\
\hline \multirow{5}{*}{$\begin{array}{l}\text { Segundo } \\
\text { freezeout }\end{array}$} & Temperatura & 38 grados & \multirow{2}{*}{$\begin{array}{l}\text { Al minuto } 5 \text { se asegura la } \\
\text { temperaura }\end{array}$} \\
\hline & Tiempo & $15 \mathrm{~min}$ & \\
\hline & $\mathrm{pH}$ & $4.00-5.50$ & \multirow{3}{*}{$\begin{array}{l}\text { Al minuto } 15 \text {, antes de la } \\
\text { transferencia a empaque }\end{array}$} \\
\hline & Alcohol cetílico & $2.50-4.50$ & \\
\hline & Apariencia & Homogénea & \\
\hline Llenado & Límites de llenado & $391,95-394,00 \mathrm{~g}$ & Al $100 \%$ de la producción \\
\hline Etiquetado & Centrado & $+/-2 \mathrm{~mm}$ & Muestreo aleatorio \\
\hline Empacado & $\begin{array}{l}\text { Materiales } \\
\text { completos }\end{array}$ & $\begin{array}{l}0 \text { Faltas de material } \\
\text { de empaque }\end{array}$ & Al $100 \%$ de la producción \\
\hline
\end{tabular}

Figura 13. Parámetros críticos en la fabricación de acondicionador Elaboración propia

\section{CONCLUSIONES}

Al terminar esta investigación y con los resultados obtenidos, se pudo concluir que el objetivo principal de este trabajo se ha cumplido. La propuesta presenta fundamentos estadísticos y de campo factibles, es decir, las empresas manufactureras de shampoo y acondicionador pueden reducir el número de muestras a fin de reducir el impacto financiero manteniendo la rastreabilidad y la estabilidad de las variables críticas de producción, siendo esto último la base de la discusión de este trabajo.

Esta conclusión se basa en los resultados descritos en la etapa financiera y en la etapa de calidad. En la etapa financiera, se comprobó tener un diferencia en el número de botellas tomadas de la línea de producción para su muestreo, de 315 botellas a 3 botellas, representando una reducción de 1,02 \% en un lote de shampoo y un 1,01\% en un lote de acondicionador. Esta optimización representa tener 312 botellas adicionales en la situación actual para venta directa a cliente. 
Adicional al costo obtenido de reducción de muestreos, se sugiere obtener la ganancia que se tendría con estas piezas que en la situación actual forman parte del scrap. Es decir, un costo de manufactura de la botella de shampoo de cuidado clásico de $400 \mathrm{ml}$ es de $\$ 6,30 \mathrm{MXN}$ y de $\$ 5,80 \mathrm{MXN}$ de una botella de acondicionador de $400 \mathrm{ml}$ de la versión de rizos definidos, siendo estos productos los más vendidos en el mercado mexicano, por lo que se recomienda agregar el costo de venta a cliente como ganancia adicional. Es importante mencionar que este costo de manufactura no incluye el costo logístico de transportación de la planta de producción a instalaciones del cliente, por lo que este monto se tiene que agregar al costo de manufactura para proyectar la ganancia adicional por pieza de venta.

En la etapa financiera, es posible concluir que tomando en cuenta 18 lotes diarios de producción de shampoo y 8 lotes diarios de acondicionador, se puede lograr una optimización de costos anual de muestreo por la cantidad de \$ $9691968 \mathrm{MXN}$ para shampoo y \$ $4600284 \mathrm{MXN}$ para acondicionador teniendo un total de ahorro de \$ $14292252 \mathrm{MXN}$ en toma de muestra. Esta cantidad anual es confirmada si el número de lotes se mantiene y el costo de manufactura es el mismo. Se sabe que este último costo pude variar según los insumos de proveedores y las negociaciones que se tengan.

Para sustentar la reducción de unidades para el muestreo, fue necesario explicar ampliamente la etapa de calidad donde la validación de un producto es el paso básico para soportar esta recomendación. La validación se debe realizar cuando existe alguna modificación, cambio o nuevo producto en la planta a través de estudios de R\&R. Esta investigación mostró la validación del proceso de ambos productos asegurando que los parámetros de las variables críticas de proceso se cumplen y se mantienen en el tiempo, tomando en cuenta el proceso de granel y el proceso de empaque de tres lotes de producto terminando consecutivos manufacturándolos en diferentes turnos con la finalidad de que los operadores supieran controlar las variables elegidas según la tecnología.

También se revisaron los resultados de las muestras tomadas en lotes de producción fuera en alguna variable. Se comprobó que las 315 botellas mostraron la misma variable fuera de especificación, es decir, analizar en el laboratorio 3 o 315 botellas da el mismo resultado, en este caso, que el lote se encuentra fuera de especificación y no puede ser vendido a cliente. 
Adicional a los costos extras que se han mencionado en este apartado, también se recomienda proyectar los ahorros de insumos de los reactivos de laboratorio y la productividad en horas hombre de los analistas y de las personas de producción que toman las muestras en la línea de producción.

Como se ha mostrado en todo el trabajo, pensar en diferentes opciones de los métodos tradicionales permite establecer nuevos procesos de liberación de producto final sin poner en riesgos el desempeño del producto al asegurar que las características que busca el consumidor en el producto se mantienen a lo largo de la vida útil del producto.

\section{REFERENCIAS}

Canipec. (2017). ¿Quiénes somos? Recuperado de http://canipec.org.mx

Coleman, L. (2015). Advanced Quality Auditing: an auditor's review of risk management, lean improvement, and data analysis A. S. f. Quality (Ed.) Recuperado de https://app.knovel.com/hotlink/pdf/ id:kt00ULZ1PA/advanced-quality-auditing/process-capability-cp

Martínez, T. (4 de diciembre de 2015). PIB de estados del Bajío, con el mayor crecimiento en una década. El Financiero.

Moguel, E. L. (2014). Memoria Estadística 2014. Recuperado de http://www.canipec.org.mx/woo/xtras/Presentaci\%C3\%B3n\%20 ejecutiva_anuario\%202014.pdf

Montgomery, D. (2004). Control estadístico de la calidad (2004 ed. Vol. XXI). México: Limusa.

Pilotzi, J. (2018). Pese a la inseguridad, la región de El Bajío crece. Recuperado de https://www.forbes.com.mx/pese-a-inseguridadla-region-de-el-bajio-crece/

Salud, S. d. (1995). Norma Oficial Mexicana NOM-141-SSA1-1995 Bienes y servicio. Etiquetado para productos de perfumería y belleza preenvasados. Recuperado de http://www.cofepris.gob. $\mathrm{mx} /$ Marco Juridico/noms/141ssa1.pdf

Sánchez Pantoja, S. E. (5 de julio del 2017). Proceso de shampoo y acondicionador/Interviewer: M. d. C. R. Guerrero. 
Silva, S. I. A. (2012). Cuánto cuestan los productos para el cuidado personal. Recuperado de http://www.profeco.gob.mx/encuesta/ brujula/bruj_2012/bol218_pcuidpers.asp

Vega Rangel, R. (1 de abril de 2016) Auditoría de Cosméticos/ Interviewer: M. d. C. R. Guerrero.

Vega Rangel, R. (18 de mayo de 2016) Producción en Procter \& Gamble/ Interviewer: M. d. C. R. Guerrero. 